MATEC Web of Conferences 36, 02001 (2015)

DOI: $10.1051 /$ matecconf/ 20153602001

(C) Owned by the authors, published by EDP Sciences, 2015

\title{
Electrostatic imaging for the Turin-Shroud Man
}

\author{
Francesco Lattarulo \\ Department of Electrical and Information Engineering, Polytechnic of Bari, 70125 Bari, via Orabona 4, Italy \\ francesco.lattarulo@poliba.it
}

\begin{abstract}
This paper is especially aimed at claiming the electrical imaging as the most eligible transfer mechanism, than among others, in relation to the undistorted (to some extent, to be quite specific) body features of the Turin Shroud Man. Explaining the good quality of an on-plane electrostatic rendering turns out to be a rather hard exercise because of the refined composition of arguments that the subject carries along behind it. These need careful attention, as that paid to the greatest possible extent in the present treatment, in order to set up an indispensable theoretical background preparatory to any future experimental validation. We are very hopeful that this outcome can be reached with the stipulation that the exposed body is embedded in a suitably pre-ionized airspace. Accordingly, a suitable radon-originated air ionization scenario, internally to a closed space, is envisaged and discussed in the present stage of the investigation. The radon concentration needed to trigger and control interspersed corona activity is carefully examined. This activity results in a typical accelerated-aging surface agent for fabric and other dielectric materials. The given physicochemical degradation is here assumed as being directly implied, under prescribed conditions, even in the special image formation pertaining to the Shroud of Turin. Perhaps, the most striking prediction made in the paper is that, under tectonic conditions which are usually met in a number of radon-prone areas, radon overconcentrations can attain and exceed the level related to the discharge onset. Interestingly, this is also the case for the Old City of Jerusalem as a place intimately connected to Turin Shroud studies.
\end{abstract}

\section{Introduction}

Detecting the physicochemical mechanism responsible for formation of a human image on a piece of fabric is resulting in the biggest challenging research area among those pertaining to the Turin Shroud (TS). The huge amount of reasoned observations (abbreviated with Obs. from now on, Ob. in the singular) collected thus far [1] could be seen as something helpful for the investigation. For the sake of argument, they are summarised in simpler form as follows

a) the envelope is permitted to be somewhat defective in taking a tight-fitting form without compromising the image impression on both the frontal and dorsal surface areas facing the inner Turin-Shroud Man (TSM);

b) the impression is extremely superficial (order of some tenths of micrometres, thus only involving a restricted number of fibres of the spun yarn) and disappears in the confined bloodstained whereabouts;

c) coloration is a sort of accelerated-aging signature where oxidation, dehydration and mechanical weakness especially affect the thin primary cell wall of the image fiber;

This is an Open Access article distributed under the terms of the Creative Commons Attribution License 4.0, which permits unrestricted use, distribution, and reproduction in any medium, provided the original work is properly cited. 
d) the micro-scale pattern looks, roughly speaking, like a monochrome stipple rendering, with the specification that a number of fibres composing the linen thread are colored, while those disposed side-by-side with the former are not;

e) the above discrete pattern turns into a smoothed out macro-scale human image whose 3D features have been transferred with negligible distortion.

The above set of evidences are here assumed as being reasonable conditions to be cumulatively met in order that an imaging phenomenology can be taken into due account. In the author's opinion, there are convincing arguments leading to identify corona activity (CA) in air with an appropriate imaging agent, without seeking as much valid competitors. By the way, Ref.[2] is recommended for an updated and exhaustive state of the art on this rather involved subject. It is worth noticing that CA is responsible for a sort of accelerated aging on the exposed side of insulating materials (see, for example, [3]) with striking similarities with those indicated in the above list with letters c) and d). That said, the fundamental working hypothesis here adopted is that a CA proliferation surrounding the TSM has been triggered by ambient radon's exhalation and concentration. This is about a reasonable and self-consistent natural phenomenology and, as such, does not require a supernatural cause. Nevertheless, the fact remains that the human image impressed on the TS looks preserved from marks of body's decomposition or dragging.

A previous description of preliminary circumstances applicable to the TS image formation is furnished in [4]. The envisioned scenario, here carefully re-examined, is delimited by a natural or man-made cavity positioned onto a mountainside in East Jerusalem (the Old City area). This zone, previously categorised as a radon-prone one [5], is part of the Jordan Rift Valley where radon sources are underground veins, or outcrops, mainly made of phosphorite. The radon exhalation and accumulation in air filled and closed spaces can only be preceded by below ground emanation along unpredictable routes up to the surface [6]. A rift appears typically fractured somewhere as a result of long-termed geologic history. Anomalous radon emanation is especially promoted in those damaged locations in association to tectonic activity [7].

Note that CA generation somehow alternative to ambient radon, and pertaining to TS studies, are possible only as a matter of principle because the common difficulty lies in meeting the set of Obs. a)e) altogether. For instance,

- $\quad$-hole theory is a fascinating late proposal that seems still under discussion in the geological scientific community (see [8] and related papers);

- $\quad$ neutron emission theory seems not to give specific arguments in relation to the expected imaging performances of the joined electromagnetic field [9];

- high-powered laser can only give rise to confined marks similar to those reported in Obs. c) and d). In other words, reproducing an integral TSM results in a purely theoretical expectation [10];

- low-powered d.c. high-voltage systems on one hand allow entire TSM-like imprinting to be obtained with fairly good reproduction of micro-scale features and with a reasonably lowenergy consumption; on the other, the image looks cylindrically distorted and impressed on the outer, instead of the inner, side of the piece of fabric corresponding to the TSM frontal region. Furthermore, the dorsal image has not been obtained so far [11].

In view of the main subject of this paper regarding electrical imaging, it is worth adding a few words on the last bullet point. The delivered low energy depends on the fact that purely glow modes, thus deprived of accidental energetic streamers, was triggered once the applied d.c. voltage exceeded the CA onset level. Therefore, a nearly no-load high voltage was applied to the electrode system in order for the low-powered impressive effect to be superficial and according to Obs. b)-d). The object under test, namely the conducting body mimicking a human subject, was suspended above ground and raised at a suitable potential according to a standard procedure for high-voltage testing. Optional electrode setups, in principle to be preferred for improving the macro-scale image features, prove to be unpractical because they would require d.c. onset voltages far larger than those attainable in standard 
high-voltage labs. Indeed, the raised drawback which consists in image location on the upper side of the frontally positioned piece of linen, thus evoking a sort of barrier effect [12], [13], can be viewed as a problem to overcome in the near future. The same also applies to the image absence on the dorsal side of the cover, in the sense that no further efforts was deliberately made at that testing stage to get the mockup's pair of side images all at once.

\section{Radon decay related image mechanism. Preliminary remarks}

The Dirichlet principle applied to the E-field domain of volume V reads [14]

$$
D=\int_{V}\left(\frac{1}{2} E^{2}-\varphi \frac{\rho}{\varepsilon_{g}}\right) d v
$$

where D is often termed Dirichlet energy. Notations $\varphi, \rho$ and $\varepsilon_{0}$ respectively indicate the potential of the field $\mathbf{E}=-\operatorname{grad} \varphi$, the volumetric density, say, of a motionless space charge and the dielectric permittivity of a gaseous medium. Indeed, preference is here expressed for the alternative version

$$
D=\int_{V}\left(\frac{1}{2} E^{2}-\varphi d i v E\right) d v
$$

playing on the fact that Eq.(1) clearly evokes Gauss' law $\varepsilon_{0} \operatorname{div} \mathbf{E}=\rho$. The minimization condition for the functional $\mathrm{D}$ implies that the electrostatic energy stored in the volume $\mathrm{V}$ is as small as it can be. Eq.(2) can be adopted as it stands also when the charged fluid is set in a steady motion (this remark is important in the present investigation, because CA is sustained by a slowly moving space charge). In fact, the stationary $\mathbf{E}$-field of a steady flow of charges is a potential field as so is an electrostatic Efield. When the ion drift is presumably subsonic, then $\operatorname{div} \mathbf{v}=0$, with $\mathbf{v}$ being the local velocity [15]. In turn, even $\operatorname{div} \mathbf{E}=0$ because $\mathbf{v}=\mathrm{kE}$, with $\mathrm{k}$ standing for ion mobility. It is now clear that the optional Eq.(2) is especially useful to ultimately realize that

$$
D=\int_{V} \frac{1}{2} E^{2} d v
$$

and

$$
w_{E}=\frac{1}{2} \varepsilon_{0} E^{2}
$$

Here, $\mathrm{w}_{\mathrm{E}}$ represents the volumetric density of the electrodynamic energy $\mathrm{W}_{\mathrm{E}}=\varepsilon_{0} \mathrm{D}$ stored in the volume V, with D obeying Eq.(3). Eqs.(3) and (4) are in tandem much more meaningful that they seem at first sight. They point out that no space-charge accumulation is permitted $(\operatorname{grad} \rho=0)$ along any given $\mathbf{E}$-field fluxline (or $\mathbf{v}$-field streamline, indifferently) for a charged subsonic flow. In fact, the subsonic flow of a physically compressible fluid is incompressible.

The distributed field source $\rho$ pertaining to this investigation identifies with the space charge produced by ionizing radiation. The above theoretical remarks importantly inform that $\rho$ tends to hold constant irrespective of being the ionized gaseous fluid at rest or in motion. The stable value of this space-charge distribution is the result of a balance between ion generation and ion-ion recombination processes. Let a given air filled room be subject to below-ground sourced exhalation and concentration of radon gas along with its decay-chain airborne products. An unsophisticated formula for $\rho$ almost seems to be

$$
\rho=e \sqrt{\left(r_{u}-\frac{1}{r_{u}}\right) \frac{R_{E}}{r_{E} \alpha}}
$$

for evaluating the amount of net space density $\rho$ of small ions (these form the population of charge carriers that can easily put in motion). In Eq.(5), e $=1.610^{-19} \mathrm{C}$ and the ratio $\mathrm{R}_{\mathrm{E}} / \mathrm{r}_{\mathrm{E}}$ are, respectively, for the elementary charge and ion pairs that are produced. This dimensionless factor establishes a link between the energy $R_{E}$ collectively released by the involved radioactive elements and that, notably $r_{E}$, 
required to produce an ion pair. The multiplicative coefficient associated to $r_{E}$ is the recombination coefficient $\alpha=1.610^{-6} \mathrm{~cm}^{3} / \mathrm{s}$ (see one step further as regards the parameter $r_{u}$ ). Eq.(5) is a specialised and novel steady-state version of a primal time-dependent governing equation, the latter routinely found elsewhere [6], [7], for the charge concentration. What is something new in that formula is the quantity in parenthesis, under the square root. This quantity takes into account that the difference between the positive $\mathrm{n}^{+}$and negative $\mathrm{n}^{-}$number densities of the implied small-ion species corresponds to the ratio $\rho /$ e. The ultimate structure of Eq.(5) has been developed with a view to the elsewhere referred to as unipolarity coefficient $r_{u}=n^{+} / n^{-}>1$ (a value comprised between 2 and 3 is typical) [16]. In comparison to the positive ions, the larger mobility of the negative counterpart accounts for the exceeding fractional quantity of these ions destined to be attached to, and neutralised by, the comparatively immobile aerosol particles. This unbalanced category of positively charged ions escaping recombination, thus represented by $\rho$ in Eq.(5), is especially detectable in closed environments. Conversely, as it is the usual case outdoors, $\mathrm{n}^{-}$so significantly approaches $\mathrm{n}^{+}$that Eq.(1) tends to vanish. An electrostatic case study will be taken into account in the ongoing description to explain the undistorted image appearing on the inner side of an unfold insulating fabric. Of course, assumption is made that the impressing mechanism performed after the fabric was wrapped around a corpulent conductor. This body is assumed as being electrically discharged and suspended in a gap where a uniform, or nearly so, electric field $\mathbf{E}$ is applied.

\section{Electrostatic scenario}

According to Eq.(5), only a fractional amount of small ions, notably the unpaired ones of positive polarity, are permitted to take part in the above definition of $\rho$, whereas the majority of this light airborne category can be neglected. In fact, the latter is assumed to accommodate pairs of oppositely charged carriers so positioned at short distance each other that a smoothed out neutrality may be claimed. This description is distinctive of a calm-air situation, exactly one subject to unimportant forced transport phenomena. For reasons of computational simplicity, $R_{E}$ in Eq.(5) is made formally dependent only on concentration $\mathrm{C}$ of the referential isotope ${ }^{222} \mathrm{Rn}$. In fact, all the decay-chain products (progeny) are indeed taken into account through a sum of normalised individual concentrations. Accordingly,

$$
R_{E}=\xi^{\prime} 10^{6} \mathrm{C}
$$

The amenable version of Eq.(6), in comparison to those available in the current literature (see [6] and [17] as a pair of examples), are not detrimental for the ongoing treatment. Provided $\mathrm{R}_{\mathrm{E}}$ and $\mathrm{C}$ are customarily expressed in $\mathrm{eV} /\left(\mathrm{cm}^{3} \mathrm{~s}\right)$ and $\mathrm{Bq} / \mathrm{m}^{3}$, respectively, then the dimensional proportionality factor $\xi$ ' can assume unpredictable values, however of the order of 10 . Note that this quantity nearly reduces to one half, or thereabouts, whether the contributions of the progeny is, say, inexcusably neglected. Consistent with the dimension previously established for $\mathrm{R}_{\mathrm{E}}$, the known value of the parameter $\mathrm{r}_{\mathrm{E}}$, incorporated in Eq.(5), can be set equal to $32 \mathrm{eV}$.

It has previously been remarked that the charge density $\rho$ can be assumed as being uniformly distributed throughout the closed room under examination. Indeed, the above distribution can even be restricted to a fractional amount of the entire room. In any case a simplified relationship, between this source and the generated background E-field, can be obtained if the field is also uniform. Note that this field is in general non-uniform, in spite of the uniformly distributed source represented by the constant value of $\rho$. However, there is a rather large subspace in the room where the field becomes nearly uniform. This airspace portion is centered on and extended just above the room floor where

$$
\boldsymbol{E}=\xi^{\prime \prime} \rho 10^{11} \boldsymbol{n}
$$


can be written [18]. This very simple formula derives after some mathematical manipulations, here omitted for brevity sake, involving a basic integral equation reported elsewhere [19]. In Eq.(7), E and $\rho$ are expressed in $\mathrm{V} / \mathrm{m}$ and $\mathrm{C} / \mathrm{m}^{3}$, respectively; $\mathbf{n}$ is a downwardly directed unit vector and $\xi^{\prime \prime}$ is a dimensionless proportionality factor of the order of some unities. This factor takes into account the actual shape of the floor and the superposition of the space-charge image. The latter is imaginatively positioned in the lower half-space, due to the presence of an equipotential ground plane on which the floor itself lies. Eq.(7) tacitly assumes that the field points are distant just enough from the room's peripheral walls and ceiling for this formula to be safely applied.

Let the object be now introduced in the space charge region governed by Eq.(7). What importantly happens is that $\rho$ and $\mathbf{E}$ regain an unperturbed uniform distribution after a brief transient time. Simple theoretical considerations involving E-flux conservation lead to ascertain that on the surface of the covered conductor the electric field reduces to the transversal component $\mathbf{E}_{\mathbf{T}}$ of the applied $\mathbf{E}$-field. Therefore,

$$
E_{T}=\xi \rho 10^{11} \cos \theta
$$

where $\theta$ is of course the angular deviation of the component $\mathbf{E}_{\mathrm{T}}$ with respect to the vertically applied total E-field.

Rearranging Eqs.(5)-(8) and remembering that the prerequisite for any corona activity to be triggered is that $\mathrm{E}_{\mathrm{T}} \geq \mathrm{E}_{\mathrm{o}} \approx 10^{6} \mathrm{~V} / \mathrm{m}$, with $\mathrm{E}_{\mathrm{o}}$ denoting the corona onset strength under standard ambient conditions as regards temperature and pressure and when a rather significant humidity is taken into account (these are usual circumstances in a cave), then

$$
C \geq \xi 10^{3} / \cos ^{2} \theta
$$

can be written. This is the condition for the indoor radon concentration $\mathrm{C}$ to be met in order that CA can be triggered, and sustained, somewhere on the conductor surface. In Eq.(9), $\xi$ is of the order of some unities provided $\mathrm{C}$ remains expressed in $\mathrm{Bq} / \mathrm{m}^{3}$. As a consequence, the condition shown in Eq.(9) can episodically be respected during abnormal radon exhalation. With the stipulation that $\theta$ is close to zero in a given surface point, the CA is expected to appear there because $\mathrm{C}$ can attain (and exceed), say, the order of $10^{4} \mathrm{~Bq} / \mathrm{m}^{3}$, a typical value that is found in several radon-prone areas of Israel [5], even in that Jerusalem district of special interest in this investigation. Similar C-levels are repeatedly monitored, as an example, in the close below-grade space of a specified dwelling [20]. This is located in an Italian radon-prone area, where TS-like images are naturally reproduced.

\section{Image transfer}

Abnormal ambient ionization is that dictated by the pair of values $\left(R_{E}, r_{u}\right)$ and, in turn, by $\rho$ through Eq.(5). As a consequence, Eq.(9) establishes location and extension of the CA-related aging. In fact, the body image surrounding an overstressed point, one of those where $\theta=0$ can be set, fully turns into the non-body image as soon as the odd-changing angle $\theta$ departs, depending on the body shape, somewhat from zero. By the way, that is a convincing argument for explaining why the image disappears even if the body-fabric contact is ensured (see, in particular, Ob. a) in Sect. 1). A remarkable TS feature is that the sum of the confined body-image surface areas is invariably less than that given summing the linen-body contact areas, in any way that covering burial cloth might have been employed to fit the corpse.

On the other hand, the severity of the CA, the latter being identified as the root cause for the impressed image (see details in [4]), is mediated and controlled by Eq.(8). It could be said that the changing concentration of the monochrome stipple pattern covering the body image areas (see Ob. d) 
in Sect. 1) depends on Eq.(8). Therefore, Eqs.(8) and (9) are crucial in accounting together for the undistorted transfer of the TSM features on the TS when this is laid down on a plane.

But if that is overcautiously estimated to be still far from enough, some supplementary arguments do concur in making Eq. (8) consistent with Obs. d) and e) in Sect. 1. The more the CA severity increases proportionally to $\mathrm{E}_{\mathrm{T}}(\theta)$, the more the monochrome point marks tend to concentrate. This performance accounts for the colour-gradated macro-scale negative pattern previously remarked in $\mathrm{Ob}$. d). It is worth considering that the good quality object rendering, raised in that observation, is to be essentially attributed to Eq.(8) because of its perfect analogy with Lambert's $\cos -\theta$ law with index 1 . This vision theory law is the prerequisite for a generically shaped 3D object to be successfully rendered, say, by a planar chiaroscuro. Accordingly, $\theta$ rather represents reflectance's angular deviation, on the surface of an illuminated scene, with respect to the orientation of a uniformly illuminating irradiance. Such a simple formula holds in optics with the stipulation that the surface of the exposed object is a diffusely reflecting rough one, as it is largely the case in most applications [21]. Also consistent with the subject under examination is that the irradiance is collinear with the line of sight of an idealized distant observer. As Eq.(8) differently reads

$$
E_{T}(\theta)=E_{T}(\theta=0) \cos \theta
$$

because $\mathrm{E}_{\mathrm{T}}(\theta=0)=\xi " \rho 10^{11}$, then the electric field and irradiance mutually play the role of analogical vector quantities, according to which the specific vector $\mathbf{E}_{\mathbf{T}}(\theta=0)$ and the generic vector $\mathbf{E}_{\mathbf{T}}(\theta)$ happen to assume the counterparts of the vertically incident plane wave's irradiance and surface reflectance, respectively. By virtue of the same analogy, the laying plane for usual TS observations may be assimilated, according to a usual terminology adopted in vision theory, to the image plane.

To better appreciate the value of this electrostatic description leading to Eq.(10), consider, as a hypothetical alternative, the case in which the surface CA is triggered in a space-charge-free gap (this is the case for Ref. [4]). The given distribution law for the surface electric field somewhat departs from the structure of Eq.(10). As a visual example, consider the negative 3D-shading images of contiguous bodies forming an array of cylinders, see Figs. 4 and 5 in Ref. [4], panels b). These clearly differ to some extent from the expected rendering reproduced in the a)-labelled counterparts. The latter successfully give the perception of round cylinder because the image obeys Lambert's law through the analogical Eq.(10). Instead, the former shading is so compromised with the mutual electrostatic influence among the cylinders that they are illusorily perceived as objects that protrude too much, as though they rather were elliptic, than circular, cylinders. Roughly speaking, the space charge tends to effectively smooth out electrostatic perturbations caused by and surrounding any given exposed conductor, a performance tacitly expressed by the structure of Eq.(10).

\section{Concluding remarks}

The ultimate purpose of this paper is to bring considerable insight into the transfer mechanism of the TSM's body features. The described natural mechanism proves that the electrical imaging applied to the TS is based on physically admissible arguments. In particular, these advise that the image details are so prone to change, according to a number of previously unexploited radon-ionized ambient conditions, that a purely CA-based impressing mechanism suffices for a circumstantial explanation of the TS image. This to stress that trying to introduce additional impressing agents different from and superimposed to the CA, possibly to be exhaustive in explaining some TS image features (see, for example, Ref. [22]), turns out, in the author's opinion, to be an unnecessary intellectual effort. By the way, Eq.(10) plays a key role in giving undistorted images by virtue of the claimed analogy with the referential Lambert's cosine law for object rendering. 
For the benefit of the reader, a pertaining numerical example has been worked out and illustrated in Appendix. A comparison is also made with the case in which the episodic radon overconcentration $\mathrm{C}$ $=10^{4} \mathrm{~Bq} / \mathrm{m}^{3}$ is replaced with a smaller value, say, $10^{2} \mathrm{~Bq} / \mathrm{m}^{3}$. According to the described mechanism, it is worth considering that the imaging success only depends on specific or local quantities and are quite independent on dimensional data. This appealing issue also regards the amount of time $t_{p}$, represented by the ratio $\mathrm{w}_{\mathrm{E}} / \mathrm{R}_{\mathrm{E}}$, required to ultimately obtain electrical imaging. Rearranging Eqs.(4), (5) and (7) gives a quantity of the order of some minutes for the above ratio. The given preparatory time is therefore independent on both $\mathrm{C}$ and room dimensions. Otherwise, the time at hand is importantly dependent on standardized ambient-air parameters (through the product $\mathrm{r}_{\mathrm{E}} \alpha$ ) and the actual unipolarity coefficient (through the difference $r_{u}-1 / r_{u}$ ). In the light of this rigorous reasoning, proper account must be taken of experimental activity conducted in laboratory on scaled mockups or in natural rooms on short-sized, variously-shaped objects. Such remarks establish more defensibility, in particular, to Ref. [20].

All things considered, the success of this electrical imaging is expected being determined by the following concurring conditions

- $\quad$ radon concentrations rather exceeding the order $10^{3} \mathrm{~Bq} / \mathrm{m}^{3}$;

- natural short-lived radon invasion either way compatible with a predetermined ambient-air preparatory time;

- actual unipolarity coefficient $r_{u}$ substantially exceeding unity (this is the case for indoor calm-air conditions);

- CA onset electric field (less than $30 \mathrm{kV} / \mathrm{cm}$ ) as short as it can be, depending on ambient air parameters (especially aqueous content).

By way of final remark, the actual numerical values referred to the parameters coming into play in Appendix represent a further convincing argument on the low energies involved in the natural electric imaging here presented. The primal fact is that the energy associated to each minute avalanche of the glow discharge here called in cause can only be less than $1 \mathrm{eV}$ [23]. We are talking about a diffusedischarge mode, so that any given image point (see, again, Obs. d) and e) in Sect. 1) can rather be the effect of locally pulsating avalanches of the distributed glow. Accordingly, the image under examination only requires local energies of the order of $1 \mathrm{eV}$ per pulse, irrespective of the point under examination. As extensively reported in Refs. [3], [4] and [23], the low elemental energy accompanying the glow-mode $\mathrm{CA}$ is on one hand responsive for the superficial character of the marks raised in Ob. b) and exhaustively explains, on the other, what has been reported in Ob. c). In this respect, note that the accelerated micro-scale aging all around the image fibre is reasonably expected to occur if the surrounding interstices are filled with chemically aggressive gases. This is exactly the case for an electrical imaging which, as a matter of fact, ultimately identifies in a chemical aging mediated by the CA in air. Discharges, in fact, typically cause production of ozone and nitric acid which are overall responsible for a chemically aggressive degradation. This could easily affect the thin primary cell wall of image fibers according to a radial penetration into them. Bear in mind that CAmediated chemical by-products find easy opportunity to fill the airspaces between the surface image fibers because of the pushing action of the ionic wind usually accompanying CA [15]. No mention has been deliberately made in this micro-scale description to purely physical CA-related agents. In fact, the distributed thermal and kinetic energies accompanying a no-streamer glow-mode coronal proliferation are unimportant for aging. The above-mentioned local energy of $1 \mathrm{eV}$ is quite ineffective in directly impressing perceivable marks by erosion due to kinetic bombardment of ion particles or by some thermally induced effects [3], [23]. Metaphorically speaking, the cold CA implied in this application behaves (only in a physical, not chemical, sense) as a continuous bombardment of ping pong balls on the surface of the board: the latter however holds on with insignificant consequences. 


\section{Appendix. Explanatory Examples}

The supposed particular environmental conditions of a Jerusalem cave, extracted from literature, are here compared with more common conditions in order to show how a CA could be naturally developed.

Let the episodic radon overconcentration be $\mathrm{C}=10^{4} \mathrm{~Bq} / \mathrm{m}^{3}$, in the airspace of a closed cave positioned in a radon-prone area. According to Eq. (6), the energy released by the radioactive elements is:

$$
\mathrm{R}_{\mathrm{E}}=10 \times 10^{6} \times 10^{4}=10^{11} \mathrm{eV} /\left(\mathrm{cm}^{3} \mathrm{~s}\right)=10^{-2} \mathrm{~W} / \mathrm{m}^{3}
$$

where $\xi^{\prime}=10$ (realistically assigned) and $10^{6}$ is a dimensional quantity which takes into account the fact that the unit volume is usually referred to $C$. $R_{E}$ and $C$ are respectively expressed in $B q / \mathrm{m}^{3}$ and $\mathrm{eV} /\left(\mathrm{cm}^{3} \mathrm{~s}\right)$. According to Eq.(5), the volumetric charge density is:

$$
\rho=1.610^{-19} \sqrt{\left(2.6-\frac{1}{2.6}\right) \frac{10^{11}}{32 \cdot 1.6 \cdot 10^{-6}}} \approx 10^{-11} \mathrm{C} / \mathrm{cm}^{3}=10^{-5} \mathrm{C} / \mathrm{m}^{3}
$$

where to the unipolarity coefficient $r_{u}=n^{+} / n^{-}$is realistically assigned the value of 2.6; the elementary charge e is $1.610^{-19}$ Coulomb; the recombination coefficient $\alpha$ is typically $1.610^{-6} \mathrm{~cm}^{3} / \mathrm{s}$; the energy required to produce an ion pair, $\mathrm{r}_{\mathrm{E}}$, is $32 \mathrm{eV}$. According to Eq.(7), the background electric field is:

$$
\mathrm{E}=10^{-5} \times 10^{11}=10^{6} \mathrm{~V} / \mathrm{m}
$$

where an indicative value for $\xi "$ is 1 and $10^{11}$ is a multiplicative dimensional factor distinctive of the E- $\rho$ relationship.

According to Eq. (9), the condition for CA to appear is:

$$
\mathrm{C}=10^{4} \geq \xi 10^{3} / \cos ^{2} \theta
$$

where $\xi$ is a dimensional factor of some unities.

Therefore CA can appear somewhere in the considered environment where the angle $\theta$ is close to $0^{\circ}$. According to Eq. (4), the volumetric density of the electrodynamic energy results:

$$
\mathrm{W}_{\mathrm{E}}=0.5 \times 8.8510^{-12} \times 10^{12} \approx 4.4 \text { Joule } / \mathrm{m}^{3}
$$

where, $\varepsilon_{\mathrm{o}}=8.8510^{-12} \mathrm{Farad} / \mathrm{m}$. Therefore, the preparatory time $\mathrm{t}_{\mathrm{p}}$ results from the following ratio:

$$
\mathrm{t}_{\mathrm{p}}=\mathrm{w}_{\mathrm{E}} / \mathrm{R}_{\mathrm{E}}=4.4 / 10^{-2}=440 \mathrm{~s} \approx 7 \text { minutes }
$$

For the sake of comparison, the above values are compared in Table 1 with those pertaining to a more common case of radon overconcentration, specifically one in which $\mathrm{C}=10^{2} \mathrm{~Bq} / \mathrm{m}^{3}$. 
Table 1. Comparison between two cases of radon overconcentration.

\begin{tabular}{|c|c|c|}
\hline & $\begin{array}{c}\text { Hypothesized radon } \\
\text { overconcentration in a } \\
\text { Jerusalem cave }\end{array}$ & $\begin{array}{c}\text { More common case of } \\
\text { radon } \\
\text { overconcentration }\end{array}$ \\
\hline Episodic radon overconcentration & $\mathrm{C}=10^{4} \mathrm{~Bq} / \mathrm{m}^{3}$ & $\mathrm{C}=10^{2} \mathrm{~Bq} / \mathrm{m}^{3}$ \\
\hline $\begin{array}{c}\text { Energy released by the radioactive } \\
\text { elements }\end{array}$ & $\mathrm{R}_{\mathrm{E}}=10^{-2} \mathrm{~W} / \mathrm{m}^{3}$ & $\mathrm{R}_{\mathrm{E}}=10^{-4} \mathrm{~W} / \mathrm{m}^{3}$ \\
\hline Volumetric charge density & $10^{-5} \mathrm{C} / \mathrm{m}^{3}$ & $10^{-6} \mathrm{C} / \mathrm{m}^{3}$ \\
\hline Background electric field & $\begin{array}{c}10^{6} \mathrm{~V} / \mathrm{m} \approx 10^{6} \mathrm{~V} / \mathrm{m}(\mathrm{CA} \\
\text { onset level). }\end{array}$ & $\begin{array}{c}10^{5} \mathrm{VA} \text { feasible. } \\
\text { (CA onset level }) \quad \text { No } \\
\text { CA. }\end{array}$ \\
\hline Electrodynamic energy & $\approx 4.4$ Joule $/ \mathrm{m}^{3}$ & $0,044 \mathrm{Joule} / \mathrm{m}^{3}$ \\
\hline Preparatory time & $\approx 7$ minutes & $\approx 7$ minutes \\
\hline
\end{tabular}

Note $^{*}$ : this check is unimportant given that the simultaneous condition for $\mathrm{E}$ to be equal to or exceeding $10^{6} \mathrm{~V} / \mathrm{m}$ (CA onset value) has not been met.

\section{References}

1. G. Fanti, J.A. Botella, F. Crosilla, F. Lattarulo, N. Svensson, R. Schneider, A.D. Whanger, Int. Workshop on the Scientific Approach to the Acheiropoietos Images, ENEA Research Center of Frascati - Italy (2010).

2. G. Fanti, J. Imaging Sci. and Tech., 55, pp. 060507-1-060507-14 (2011).

3. R.J. Van Brunt, IEEE Trans. on Dielectrics and El. Ins.,1, n. 5, pp. 761-784 (1994).

4. V. Amoruso, F. Lattarulo, Sci. Res. Essays, 7, pp. 2554-2579 (2012).

5. M. Shirav (Schartz), U. Vulkan, Environ. Geol., 31, pp. 167-173 (1997).

6. N. Kamsali, B.S.N. Prasad, J. Datta, in: Advanced Air Pollution, F. Nejadkoorki Ed., (InTech, Rijeka - Croatia, 2011).

7. Y. Omori, Y. Yasuoka, H. Nagahama, Y. Kawada, T. Ishikawa, S. Tokonami, M. Shinogi M.Shinogi, Nat. Hazards Earth Syst. Sci., 7, pp. 629-635 (2007).

8. F. T. Freund, M. M. Freund, EMSEV 2012, Gotemba Kogen Resort, Gotemba - Japan (2012).

9. F. Cardone, A. Carpinteri, G. Lacidogna, Phys. Lett. A, 373, pp. 4158-4163 (2009).

10. C. Grey Morgan, in Electrical Breackdown of Gases, J.M. Meek, J.D. Craggs Eds. (J. Wiley \& Sons, New York, 1978).

11. G. Fanti, F. Lattarulo, G. Pesavento, SHS WoC, 15 (2015).

12. M. Abdel-Salam, H. Singer, A. Ahmed, J. Phys. D: Appl. Phys., 30, pp. 1017-1028 (1997).

13. F.V. Topalis, M.G. Danikas, Facta Univ. (NIŠ) - Ser.: Elec. Energ., 18, pp. 87-104 (2005).

14. R. Courant, Dirichlet's Principle, Conformal Mapping, and Minimal Surfaces. Appendix by M. Schiffer (Interscience, Dover Publ., N. Y., 1950).

15. F. Lattarulo, V. Amoruso, Filamentary Ion Flow - Theory and Experiment (IEEE Press-Wiley, Hoboken 2014).

16. P.M. Kolarž, D.M. Filipović, B.P. Marinković, Appl. Rad. Isot., 67, pp. 2062-2067 (2009).

17. M. S. Chandrashekara, J. Sannappa, L. Paramesh, Indian J. Pure Appl. Phys., 43, pp. 679-683 (2005).

18. V. Amoruso, F. Lattarulo, J. Electrostatics, 56, pp. 255-276 (2002).

19. E. Durand, Electrostatique, vol. 1 (Masson, Paris, 1964).

20. G. de Liso, F. Lattarulo, G. Fanti, SHS WoC, 15 (2015).

21. F. Lattarulo, V. Amoruso, Int. J. Appl. Electrom. Mech., 37, pp. 231-240 (2011).

22. A. D. Whanger, in: The Turin Shroud - Past, Present and Future, S. Scannerini, P. Savarino Eds., (Sindon-Effatà, Cantalupa, 2000).

23. M. Goldman, A. Goldman, J. Gatellet, IEE Proc.-Sci. Meas. Technol., 143, pp.11-16 (1995). 